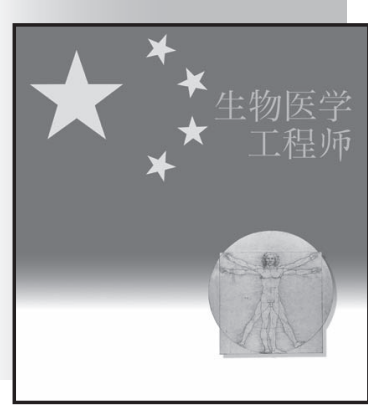

(c) WIKIPEDIA

\title{
Brain-Computer Interfaces Based on Visual Evoked Potentials
}

\author{
Feasibility of Practical System Designs
}

BY YIJUN WANG,

XIAORONG GAO,

BO HONG, CHUAN JIA,

AND SHANGKAI GAO

R ecently, electroencephalogram (EEG)-based braincomputer interfaces (BCIs) have become a hot spot in the study of neural engineering, rehabilitation, and brain science. In this article, we review BCI systems based on visual evoked potentials (VEPs). Although the performance of this type of BCI has already been evaluated by many research groups through a variety of laboratory demonstrations, researchers are still facing many difficulties in changing the demonstrations to practically applicable systems. On the basis of the literature, we describe the challenges in developing practical BCI systems. Also, our recent work in the designs and implementations of the BCI systems based on steady-state VEPs (SSVEPs) is described in detail. The results show that by adequately considering the problems encountered in system design, signal processing, and parameter optimization, SSVEPs can provide the most useful information about brain activities using the least number of electrodes. At the same time, system cost could be greatly decreased and usability could be readily improved, thus benefiting the implementation of a practical BCI.

\section{Brain-Computer Interfaces}

BCIs translate human intentions into control signals to establish a direct communication channel between the human brain and output devices. Among a variety of methods for monitoring brain activity, the most favorable method is noninvasive EEG. Various brain signals have been employed in the design of EEG-based BCIs, e.g., event-related potentials (ERPs) and event-related EEG changes [i.e., event-related desynchronization/synchronization (ERD/ERS)] [1]-[3]. Among the current BCIs, the system based on VEPs has been studied for a long period since the 1970s [4]. During the last few years, it has still received strongly increased attention in BCI research [5]-[14]. Studies on the VEP $\mathrm{BCI}$ demonstrate convincing robustness of system performance through many laboratory and clinical tests. The recognized advantages of this $\mathrm{BCI}$ include easy system configuration, little user training, and a high information transfer rate (ITR).

\section{Visual Evoked Potentials}

VEPs reflect the visual information-processing mechanism in the brain. According to the knowledge of brain electrophysiology,

Digital Object Identifier 10.1109/MEMB.2008.923958
VEPs corresponding to low stimulus rates are categorized as transient VEP (TVEP), and those corresponding to rapidly repetitive stimulations are categorized as steady-state VEP (SSVEP) [15]. Ideally, a TVEP is a true transient response to a stimulus when the relevant brain mechanisms are in resting states. It does not depend on any previous trial. If the visual stimulation is repeated with intervals shorter than the duration of a TVEP, the responses evoked by each stimulus will overlap each other, and an SSVEP is generated. In this circumstance, the brain is considered in a steady state of excitability. So far, both TVEP and SSVEP have already been applied in BCI research. Because of the characteristics caused by different stimuli, analysis of the TVEP is based on temporal methods such as template matching, whereas SSVEP detection is usually performed by frequency analysis, e.g., power spectral density (PSD) estimation.

\section{Present-Day VEP BCls}

In this section, we present a survey of VEP-based BCI systems. To emphasize the importance of an online system design, studies only presenting offline analysis results are not included here. To the best of our knowledge, eight groups have developed demonstrations during the past several decades [4]-[14]. Among three of the eight groups, each developed two different systems (see [6]-[10] and [12] for details). Detailed classification results of our survey are listed in Table 1. The principal design attributes include operation modality, signal recording (signals and number of channels) and users, visual stimulus (stimulus display and number of targets), and signal processing (information encoding and decoding). The listed references represent the systems designed with the attribute subclasses.

\section{Operation Modality}

According to the necessity of employing activity in the brain's normal output pathways to generate brain activity, BCIs are divided into two classes: dependent and independent [1]. The VEP system based on gaze detection falls into the dependent class. The generation of the VEP depends on gaze direction controlled by motor activity of extraocular muscles. Therefore, this BCI is inapplicable for people with severe neuromuscular disabilities, who may lack extraocular muscle control. Nevertheless, because of the reliability and practicability of the 


\section{Even for the groups that have realized assistive device control, many of them remain at the \\ demo stage in laboratories.}

system, all eight groups have designed similar systems employing gaze detection as the basic principle [4]-[11], [13], [14]. In addition to amplitude modulation by gaze control, recent neuroscience studies on visual attention also reveal that the VEP can also be modulated by spatial attention and feature-based attention independent of neuromuscular function [16]-[18]. These findings make it possible to implement an independent BCI based on attentional modulation of VEP amplitude. Up to now, one research group developed an independent VEP-based BCI called the visual-spatial attention control (V-SAC) BCI employing visual spatial attention to self-regulate amplitude of SSVEPs elicited by two flicker stimuli on the left and right sides of a screen [12]. Compared with the dependent type, this attention-based BCI needs more subject training and has a much lower ITR. In real-life application, the dependent type is more practical for most users. Thus, the dependent VEP-based BCI is more attractive than the independent type in current BCI research.

\section{Signal Recording and Users}

Data recording in these systems differs in signals and electrodes. Both EEG and electrocorticogram (ECoG) have been used in VEP-based BCIs. In the brain response interface (BRI) system, an epidural electrode strip implanted over the visual cortex was used in an amyotrophic lateral sclerosis (ALS) patient [5]. All the other systems use noninvasive scalp EEG electrodes. When compared with ECoG, although the EEG signal has a low signal-to-noise ratio (SNR), it is still more acceptable to the users because of its noninvasiveness. The number of electrodes can be categorized as two electrodes and multiple electrodes (4-12 electrodes). Although multichannel data are more reliable for data analysis, considering the practicability, most systems are designed with few electrodes. The $\mathrm{O} 1$ and $\mathrm{O} 2$ electrode positions of the international 10-20 system are widely used. The bipolar electrode placement has been applied in some systems to obtain a cleaner signal by canceling common background activities [6], [11].

Most systems are in the stage of laboratory demonstration with healthy volunteers as the subjects. Toward clinical application, two systems have been tested with patients. The BRI system was successfully applied to help an ALS patient to operate a word-processing program [5]. Also, in our previous study, the real-life application of an SSVEP-based BCI was investigated on patients with spinal cord injury (SCI). The reason for performance decrease from the laboratory test to the clinical test was discussed in [9]. In the future, much more work is required to make the system more robust in daily-life applications for the motion disabled.

Table 1. Classification of online VEP-based BCI systems.

\begin{tabular}{|c|c|c|c|}
\hline Design Attribute & Attribute Subclass & $\begin{array}{l}\text { References with } \\
\text { Attribute Subclass }\end{array}$ & $\begin{array}{l}\text { Group } \\
\text { Counts }\end{array}$ \\
\hline \multirow[t]{2}{*}{ Operation modality } & Dependent & $(4)-(11),(13),(14)$ & 8 \\
\hline & Independent & (12) & 1 \\
\hline \multirow[t]{2}{*}{ Signals } & EEG & $(5)-(14)$ & 7 \\
\hline & ECoG & (4) & 1 \\
\hline \multirow[t]{2}{*}{ Number of channels } & 2 (e.g., O1 and O2) & $(6)-(10),(12),(14)$ & 4 \\
\hline & Multiple & $(4),(5),(11),(13)$ & 4 \\
\hline \multirow[t]{3}{*}{ Users } & Healthy volunteers & $(4),(6)-(8),(10)-(14)$ & 8 \\
\hline & Patients: ALS & (5) & 1 \\
\hline & $\mathrm{SCl}$ & (9) & 1 \\
\hline \multirow[t]{2}{*}{ Stimulus display } & LCD/CRT & (5)-(7), (9), (10), (12)-(14) & 6 \\
\hline & Flashtube/LED & $(4),(8),(11)$ & 3 \\
\hline \multirow[t]{2}{*}{ Number of targets } & Single & $(4),(6)$ & 2 \\
\hline & Multiple (2-64) & (5), (7)-(14) & 7 \\
\hline \multirow[t]{3}{*}{ Information coding } & TVEP & (4) & 1 \\
\hline & SSVEP: †SSVEP & (5), (14) & 2 \\
\hline & fSSVEP & $(6)-(13)$ & 5 \\
\hline \multirow[t]{4}{*}{ Information decoding } & Temporal waveform & (4) & 1 \\
\hline & Cross-correlation & (5) & 1 \\
\hline & Phase coherent detection & (14) & 1 \\
\hline & PSD analysis & $(6)-(13)$ & 5 \\
\hline
\end{tabular}




\section{Reducing the number of electrodes in $\mathrm{BCl}$ systems is a critical issue for the successful development of clinical applications of $\mathrm{BCl}$ technology.}

\section{Visual Stimulator}

The visual stimulator commonly consists of flickering targets in the form of alternating colors or a reversing checkerboard. In the VEP-based BCIs, the CRT/LCD monitor or flashtube/lightemitting diode (LED) is used for stimulus display. Among all the groups, six groups use a monitor to display visual stimulation, whereas three use a flashtube or LEDs. A computer monitor is convenient for target alignment and feedback presentation through programming, but for a frequency-coded system, the number of targets is limited because of the refresh rate of a monitor. Therefore, the LED stimulator is preferable for a multiple target system. The flickering frequency of each LED can be controlled independently by a programmable logic device. Using such a stimulator, a 48-target BCI was reported in [8]. In addition, the LED stimulator is more suitable for composing a portable system that does not depend on a computer. A wearable stimulator can significantly improve the practicability of VEP-based BCIs.

Among all the systems, the number of stimulation targets varies between one and 64 , which leads to a range of system performance. Generally, a system with more targets can achieve a higher ITR. For example, in tests of the 13-target and two-target systems, the subjects had an average ITR of 43 and $10 \mathrm{~b} / \mathrm{min}$, respectively [9], [10]. However, because a stimulator with more targets is also more exhausting for users, the number of targets should be considered according to a tradeoff between system performance and user comfort. In addition, the optimal number of stimuli also depends on the usable bandwidth of SSVEPs, which is subject specific [9]. The extended use of the frequencies in which the SSVEPs have low SNRs will not increase but decrease the ITR.

\section{Signal Processing: Information Coding and Decoding} According to the VEP signals used for information coding, current VEP BCIs fall into two groups: TVEP and SSVEP. The first group uses TVEP to detect gaze direction. Spatial distributions of TVEPs elicited by a stimulus located in different visual fields were used to identify visual fixation by Vidal in the 1970s [4]. The SSVEP is used by the other group as the communication medium. According to the approach for information coding, the SSVEP-based BCIs can be further divided into subgroups of temporal coding and frequency coding. To facilitate presentation, we refer to them as tSSVEP and fSSVEP, respectively. The BRI system designed by Sutter is a tSSVEP-based BCI [5]. The occurrence time of a sequence of VEPs was used to label a stimulation target. In other words, different targets evoke tSSVEPs identical in shape of waveform but different in time of occurrence. Another tSSVEP BCI is the phase-coded system designed by Kluge et al. with two stimuli at a single frequency but different phases to elicit tSSVEPs [14]. The other SSVEP BCIs utilize the fSSVEP to encode information. One or more frequency- coded stimulation targets are used to elicit fSSVEPs. During system operation, the amplitude of the fSSVEP is regulated by gaze or spatial attention.

The approaches for information decoding depend on the protocol of coding. Feature extraction of the TVEP is based on waveform detection in the temporal domain [4]. Similarly, a template-matching approach by cross-correlation analysis was used to detect the tSSVEP in the BRI system [5]. In the phasecoded SSVEP system, an approach using phase-coherent detection between the stimulation and SSVEP has been proposed [14]. In the fSSVEP-based BCIs, PSD analysis is most widely used [6]-[13]. After PSD estimation, the frequency components at the fundamental and harmonic frequencies are commonly used as features for classification. It must be mentioned that the analysis of the TVEP and tSSVEP needs accurate time triggers from the stimulator, whereas they can be omitted in power-based detection of the SSVEP.

\section{Practical System Designs}

With the VEP-based BCIs discussed in this article, many studies have been performed to implement and evaluate demonstration systems in laboratory settings; however, the challenge facing the development of practical BCI systems for real-life applications needs to be emphasized. According to the survey made by Mason et al., existing BCI systems could be divided into three classes: transducers, demo systems, and assistive devices [19]. Among the 79 BCI groups investigated, ten have realized assistive device (13\%), 26 have designed demonstration systems (33\%), and the remaining 43 are only in the stage of offline data analysis (54\%). Even for the groups that have realized assistive device control, many of them remain at the demo stage in laboratories. In other words, there is still a long way to go before BCI systems can be put into practical use. However, as an emerging engineering research area, if they remain in the laboratory for scientific exploration, their influence on human society will certainly be limited. Thus, the feasibility for practical application is a serious challenge in the study of BCI systems. A practical BCI system must fully consider the user's human nature, which includes the following aspects.

\section{Noninvasive to Users}

An important argument of BCI users is that time is not an issue for completely paralyzed patients. In Birbaumer's research, only one of 17 ALS patients agreed to use subdural electrodes, despite the fact that implanted electrodes can significantly improve system performance when compared with scalp electrodes [20]. So far, noninvasive EEG-based BCI systems are the first choice for the majority of users, including severely paralyzed patients. Thus, extracting useful information from the scalp EEG stably and reliably is the accompanying 


\section{The recognized advantages of VEP-based BCls include easy system configuration, little user training, and a high information transfer rate.}

problem, which depends on data-recording configurations and information-processing techniques.

\section{Convenient and Comfortable to Use}

Current EEG systems use standard wet electrodes, in which electrolytic gel is required to reduce electrode-skin interface impedance. Using electrolytic gel is uncomfortable and inconvenient, especially if a large number of electrodes are adopted. First, preparation of EEG recording before BCI operation takes a long time. Second, problems caused by electrode damage or bad electrode contact can occur. Third, an electrode cap with a large number of electrodes is uncomfortable for users to wear and thus unsuitable for long-term recording. Moreover, an EEG recording system with a large amount of channels is usually quite expensive and nonportable. For all these reasons, reducing the number of electrodes in BCI systems is a critical issue for the successful development of clinical applications of BCI technology.

\section{Stable and Reliable System Performance}

Compared with the environment in an EEG laboratory, electromagnetic interference and other artifacts [e.g., electromyogram (EMG) and electrooculogram (EOG)] are much stronger in daily home life. Suitable measures should then be applied to ensure the quality of the recorded EEG. Considering data recording in unshielded environments, the use of an active electrode may be much better than a passive electrode. It can ensure that the recorded signal is insensitive to interference [21]. To remove the artifacts in EEG signals, additional recordings of EMG and EOG may be necessary, and advanced techniques for online artifact cancelling should be applied. Moreover, to reduce the dependence on technical assistance during system operation, ad hoc functions should be provided in the system to adapt to the individual diversity of the user and nonstationarity of the signal caused by changes in the electrode impendence or brain state. These functions must be convenient to use. For example, software should be able to detect bad electrode contact in real time and automatically adjust algorithms to be suitable for the remaining good channels.

\section{Low-Cost Hardware}

Although BCI is known as a high-tech application, it should be remembered that its direct users are mostly disabled persons. The system cannot be popularized if it costs too much, no matter how good its performance is. To reduce the cost, two main aspects should be considered. The first is the EEG recording equipment. It need not satisfy the requirements of a commercial EEG system but only those of the specific BCI system. The cost lies with the parameters such as the number of channels, the bandwidth, and the sampling rate. The second is the signal processing unit. For most current systems, signal processing is executed on a computer. To eliminate the cost of a computer as well as design a portable system, a digital signal processor (DSP) can be employed to construct a system not dependent on a computer.

The requirements for a BCI system to reach the stage of practical application have been listed earlier. Some of the requirements complement each other, e.g., reducing the electrode number can not only enhance the convenience of system operation but also help to decrease the system cost. Other requirements may be contradictory to each other, e.g., although using scalp EEG for signal acquisition is noninvasive, it will make signal processing more difficult because of the lower SNR. The key point of the research is to weigh the importance of various factors and overcome the bottleneck, so as to find a comprehensive solution for the problems. Although solving all of these problems within a short time seems difficult, our recent research looks promising for providing a comprehensive solution. The SSVEPbased BCI system designed by us is introduced in the next section to show its practical implementation.

\section{Practical BCI System Based on SSVEP}

By gazing at different visual targets bearing different features and located at different positions, a specific target can be selected based on its specific feature and then used to control the corresponding peripheral device. This is the basic principle of our SSVEP-based BCI. At present, it is one of the BCI systems that are most promising for practical use. Toward the aim of practical applications, great efforts have been made by us in facilitating system configuration and improving system performance. According to the practicality issues aforementioned, we focus on the two aspects of lead selection and information processing, which can significantly reduce system cost and improve system performance. In our system, only one bipolar lead on subjectspecific positions is needed to obtain the SSVEP with a high SNR. The advantages of this simple electrode layout include low-cost hardware, convenient operation, and easy calculation, which can benefit the implementation of a practical BCI. Appropriate approaches to information processing can ensure a more stable system performance. Frequency features of SSVEP harmonics and phase-locking detection between the SSVEP and the visual stimulation have been investigated in our designs of frequency-coded and phase-coded systems.

In this section, both types (frequency-coded and phasecoded) of SSVEP-based BCI designed by us are described. In tests of the system based on frequency features (dialing a telephone number), with optimized system parameters for five participants, an average ITR of $46.68 \mathrm{~b} / \mathrm{min}$ was achieved [22]. For the study of phase coding, a preliminary demonstration of the system has been designed and tested. The system can reliably classify SSVEP responses for six phase-coded stimuli flickering at the same frequency. For one subject, an 
accuracy of $86.7 \%$ was achieved, demonstrating the practicability of phase coding in an SSVEP-based BCI.

\section{SSVEP System Based on Frequency Feature}

Frequency coding and PSD analysis is the method employed by most SSVEP-based BCI systems. Figure 1 shows its basic diagram. Facing a number of visual spots flickering at different frequencies, the subject gazes at one of them, generating an SSVEP with specific frequency components. Figure 1(c) is the amplitude spectrum of the SSVEP evoked by the visual spot flickering at $7 \mathrm{~Hz}$. Spectrum peaks appear at $7 \mathrm{~Hz}, 14 \mathrm{~Hz}$ (second harmonic), and $21 \mathrm{~Hz}$ (third harmonic). When the peaks are detected, the flickering spot the subject is watching can be identified, and the corresponding command can then be executed.

The SSVEP BCI based on frequency coding seems to be rather simple in principle, but a number of problems (such as selection of electrodes and stimulating frequencies, algorithm of feature extraction, and threshold setting) have to be solved during its implementation. Among them, lead position selection and frequency feature extraction are the most important [7]-[9]. As mentioned previously, because of the difference in the subject's physiological conditions, a preliminary experiment should be carried out for a new user to set the subjectspecific optimal parameters.

\section{Lead Position}

To get SSVEPs with a high SNR using the least number of electrodes is the goal of lead selection. Only one bipolar lead is chosen as the input in our system. From physiological knowledge, VEP can be recorded with maximum amplitude at the occipital region. So, the electrode giving the strongest SSVEP, which is generally located in the occipital region, is selected as the signal channel. The location of the reference channel is searched under the following considerations: the amplitude of the SSVEP in it should be lower, and its position should lie in the vicinity of the signal channel so that the noise component in it is similar to that in the signal channel. A high SNR can then be gained when the potentials of the two electrodes are subtracted. Figure 2 shows an example of a significant enhancement of SSVEP SNR derived from this lead selection method. Most spontaneous background activities are eliminated after the subtraction, whereas the SSVEP component is retained. Details of the method can be found in [9]. According to our experience, although the selection varies between subjects, once it is selected, it is relatively stable with respect to time. This finding makes the lead selection method feasible for practical BCI application. For a new subject, the multichannel recording needs to be done only once for optimization of lead position.

\section{Frequency Feature}

Because of the nonlinearity during information transfer in visual system, strong harmonics may often be found in the SSVEPs. Muller-Putz et al. investigated the impact of using SSVEP harmonics on the classification result of a fourclass SSVEP-based BCI [11]. In their study, the accuracy obtained with combined harmonics (up to the third harmonic) was significantly higher than with only the first harmonic. In our experience, for some subjects, the intensity of the second harmonic may sometimes be even stronger than that of the fundamental component (Figure 1). Thus analysis of the frequency band should cover the second harmonic and the frequency feature has to be taken as the weighted sum of their powers:

$$
\begin{aligned}
P_{i} & =\alpha P_{f_{1 i}}+(1-\alpha) P_{f_{2 i}}, \\
i & =1,2, \ldots N,
\end{aligned}
$$


where $N$ is the number of targets, and $P_{f_{1 i}}$ and $P_{f_{2 i}}$ are, respectively, the spectrum peak values of fundamental and second harmonics of the $i$ th frequency (i.e., $i$ th target), and $\alpha$ is the optimized weighting factor that varies between subjects. Its empirical value may be taken as

$$
\alpha=\frac{1}{N} \sum_{i=1}^{N} P_{f 1 i} /\left(P_{f_{1 i}}+P_{f_{2 i}}\right) .
$$

\section{SSVEP System Based \\ on Phase Feature}

In the SSVEP-BCI system based on frequency coding, the flickering frequencies of the targets are not the same. To ensure sufficiently high classification accuracy, a sufficient interval should be kept between two different frequencies and the number of targets will then be restricted. If phase information embedded in SSVEP is added, the number of flickering targets may be increased and a higher ITR should be expected. An SSVEP BCI based on phase-coherent detection was proposed by Manfred et al. [14], and its effectiveness was confirmed. However, only two stimuli with the same frequency but different phases were dealt with in their design, and the advantages of phase detection were not sufficiently shown. Besides, it can hardly be applied for online use because of its heavy computational load. Inspired by their work, we tried to further the work by designing a BCI system with stimulating signals of six different phases under the same frequency. Initial testing indicates the feasibility of this method.

\section{Stimuli Generation}

The first step of implementing a phase-encoded SSVEP BCI is its stimuli design. Spots flickering on a computer screen at the same frequency with strictly constant phase differences are required. We use the relatively stable computer screen refreshing signal $(60 \mathrm{~Hz})$ as a basic clock, and six stable $10-\mathrm{Hz}$ signals are obtained by frequency division as shown in Figure 3. They are used as the stimulating signals for the flickering spots on the screen to control the flashing moments of the spots (shown as black blocks in Figure 3). The flashing moments of the spots are staggered by one refreshing period of the screen $(1 / 60 \mathrm{~s})$. In other words, because the process repeats itself every six refresh periods, the phase difference of the flashing is strictly kept at $60^{\circ}$ (taking the flashing cycle of all the targets as $360^{\circ}$ ). Six targets flickering at the same frequency with different phases are thus obtained.

\section{Phase Information Detection}

A preliminary test was conducted by a healthy volunteer. During the experiment, the subject was asked to gaze at the six targets, and the optimized bipolar EEG signal was gathered from the electrodes located in the occipital region. The spectrum value at the characteristic frequency $\left(f_{0}=10 \mathrm{~Hz}\right)$ was calculated simply by the following formula:

$$
y\left(f_{0}\right)=\frac{1}{N} \sum_{n=1}^{N} x(n) \exp \left[-j 2 \pi\left(f_{0} / f_{s}\right) n\right],
$$

where $f_{\mathrm{s}}$ is the sampling frequency $(1,000 \mathrm{~Hz})$, and data length $N$ is determined by the length of the time window. The complex spectrum value at $10 \mathrm{~Hz}$ may be indicated on a plane of complex values as shown in Figure 4. The data length is $1 \mathrm{~s}$. The clustering property of the results is clearly shown.

It can be seen from Figure 4 that the VEP and the stimulating signal are stably phase locked. The responses evoked by stimulating signals with a phase difference of $60^{\circ}$ also have a

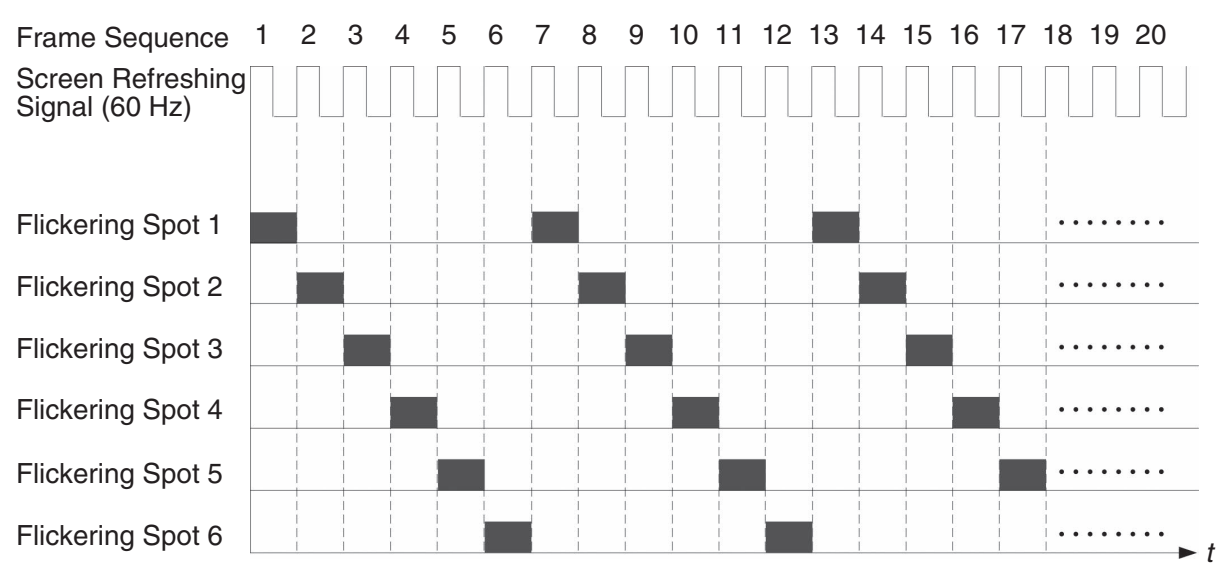

Fig. 3. Flashing sequences of the six flickering spots. The refresh rate of the monitor is $60 \mathrm{~Hz}$. The targets flicker at the same frequency of $10 \mathrm{~Hz}$ with different phases. Two adjacent flickering spots have a phase difference of $60^{\circ}$.

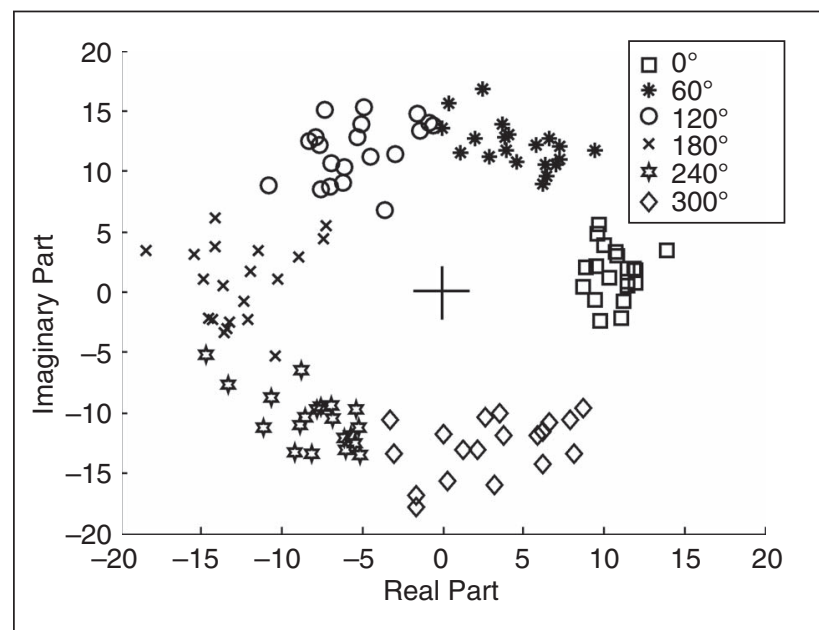

Fig. 4. Scatter diagram of the complex FFT coefficients. Horizontal and vertical axes are corresponding to real and imaginary parts of the complex spectrum values at $10 \mathrm{~Hz}$, respectively. Phase differences of stimulations are reflected by the angles of the spectrum values at the stimulation frequency on the complex plane. The cross indicates the origin of the coordinate system. 
phase difference of approximately $60^{\circ}$. This makes it possible to establish several visual targets flickering under the same frequency but with different phases so as to increase the number of targets ready for choice.

\section{Demonstration}

As an example, we used the system described above to simulate a TV controller. The flickering spots were used to control the switching (on/off), channel increase $(\mathrm{P}+)$ or decrease $(\mathrm{P}-)$, and volume increase $(\mathrm{V}+)$ or decrease $(\mathrm{V}-)$, and the spot (back) was used for returning to the previous menu to select other operations.

A simple classifier was designed based on the data from a training session before the test sessions. Average values of the FFT complex coefficients at $10 \mathrm{~Hz}$ were calculated for the SSVEPs evoked by all the phase-coded stimulations and six characteristic angles were thus found. The mean value of the characteristic angle between two adjacent categories was used as the dividing line between the two categories. Five test sessions were designated to a subject who was asked to gaze at the six targets in turn during each session. The decision was made online by the simple classifier. Among 30 targets in five sessions, 26 were correctly identified, resulting in a classification accuracy of $86.7 \%$.

\section{Outlook}

\section{Visual Stimulator}

The performance of a VEP-based BCI depends on the visual stimulator. Some recent studies present some new techniques on stimulus design. Srihari Mukesh et al. proposed a multiplefrequency stimulation method, which can be used to increase the number of selections when using a limited number of stimulation frequencies [23]. In the half-field alternate visual stimulation designed by Materka et al. [24], a stimulus in the form of left/right light sources flashing with the same frequency but alternately in time (i.e., with a phase difference of $180^{\circ}$ ) results in an enhanced SNR of the SSVEP on the bipolar lead at O1 and $\mathrm{O} 2$. In the frequency-coded system, the number of the targets will be restricted because of the limitation of the refresh rate of a monitor. In this circumstance, the LED stimulator is more flexible. Moreover, the LED stimulator provides the possibility of implementing a system based on high-frequency SSVEP ( $>20 \mathrm{~Hz}$ ). In our previous study, we demonstrated that the highfrequency SSVEP has an SNR similar to the low-frequency SSVEP [9]. (SNR is defined as the ratio of EEG power at the stimulation frequency to the mean power of the adjacent frequency bands.) Therefore, a much wider frequency band can be used as the candidate of stimulation frequency. By extending the range of stimulation frequency, we can design a system with more options to achieve a higher ITR. It can be assumed that design of a stimulator in which a flashing target can be modulated by an arbitrary modulation curve (e.g., continuously changed frequency or amplitude modulation) may further facilitate frequency selection, feature extraction, and classification.

\section{Electrode Placement}

As we know, using a small number of electrodes can reduce the cost of hardware while improving the convenience of system operation. The electrode position is very important in such a portable system with few electrodes. In most VEP-based BCIs, the electrodes are placed on $\mathrm{O} 1$ and $\mathrm{O} 2$ over the occipital region. In our system, we use a subject-specific electrode placement method to achieve a high SNR of SSVEP, especially for the subjects with strong background brain activities over the area of the visual cortex. Although we demonstrated the applicability of the subject-specific positions for some subjects in many online experiments, the stationarity of the optimized electrode positions needs further investigation. In addition, the principle of time-variant amplitude changes of VEPs and background noises in different online sessions/ experiments also has not been explored.

In the near future, more convenient electrode methods, e.g., the dry electrode [25]-[27], will be highly desirable to replace the currently used wet electrode. Under this circumstance, it is acceptable to use more electrodes to acquire more sufficient data to fulfill the detection of SSVEP signals with multichannel data analysis approaches, e.g., spatial filtering techniques described in [28] and the canonical correlation analysis method presented in [29]. An additional advantage of multichannel recording is that manual selection of electrode positions is not needed.

\section{Independent System}

The V-SAC BCI provides a feasible way to implement an independent BCI based on SSVEP in which covert spatial attention is used to make binary selection of left and right visual targets [12]. Besides spatial attention, feature-based attention is another potential protocol to construct an independent BCI system. Several studies of feature-based attention employed frequency-tagged SSVEPs to investigate this top-down modulation by higher cognitive processes. In [17], two superimposed images consisting of vertical or horizontal bars with different colors (red and green) flickering at different frequencies were presented. A similar design of stimulus was a display consisting of spatially intermingled red and blue motion dots, which were also frequency tagged [18]. Paying selective attention to one image or one population of dots and ignoring the other will enhance the amplitude of its frequencytagged SSVEP. Based on these findings, a new featureattention-based BCI can be expected. For example, a display of multicolor frequency-tagged motion dots can ideally be used to implement a multiclass BCI. Furthermore, because of the independency between spatial attention and feature-based attention, it should be possible to improve the performance of an independent VEP-based BCI by integrating them, e.g., using both types of stimulus displayed together to increase the number of targets.

\section{Acknowledgments}

This project is supported by the National Natural Science Foundation of China (Grants 60318001 and 30630022) and the Science and Technology Ministry of China under Grant 2006BAI03A17. The authors would like to thank Dan Zhang, Yixuan $\mathrm{Ku}$, and Guangyu Bin for their technical support and time in the system tests, and Steven A. Hackworth for his careful proofreading of the manuscript.

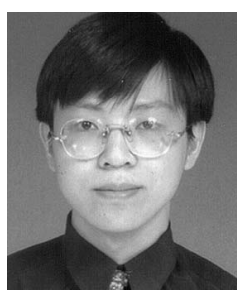

Yijun Wang received his B.E. and Ph.D. degrees in biomedical engineering from Tsinghua University, Beijing, China, in 2001 and 2007, respectively. His research interests include brain-computer interface, biomedical signal processing, and machine learning. 


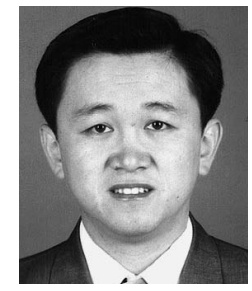

Xiaorong Gao received the B.S. degree in biomedical engineering from Zhejiang University, Hangzhou, China, in 1986; the M.S. degree in biomedical engineering from Peking Union Medical College, Beijing, China, in 1989; and the Ph.D. degree in biomedical engineering from Tsinghua University, Beijing, China, in 1992. He has been working at the Department of Electrical Engineering, Tsinghua University, since 1992. He is currently a professor with the Department of Biomedical Engineering, Tsinghua University. His current research interests are biomedical signal processing and medical instrumentation.

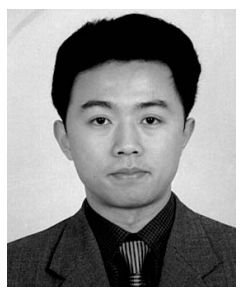

Bo Hong received his B.E. and Ph.D. degrees in biomedical engineering from Tsinghua University, Beijing, China, in 1996 and 2001, respectively. During 1999-2000, he received research training in psychophysiology at the Department of Psychology, Chinese University of Hong Kong. He has been a lecturer at Tsinghua University since 2001. On his sabbatical from 2004 to 2005, he was a visiting faculty in the Department of Biomedical Engineering and the Center for Neural Engineering at Johns Hopkins University, USA. Since 2005, he has been an associate professor with the Department of Biomedical Engineering, School of Medicine, Tsinghua University, Beijing, China. Neural information decoding and braincomputer interface are his main research interests.

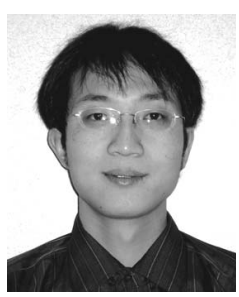

Chuan Jia received the B.E. degree in biomedical engineering from Zhejiang University, Hangzhou, China, in 2004. He is currently working toward the Ph.D. degree in the Department of Biomedical Engineering in Tsinghua University, Beijing, China. His current research is centered on brain-computer interface, particularly an applicable brain-computer interface from both the engineering and physiology perspectives.

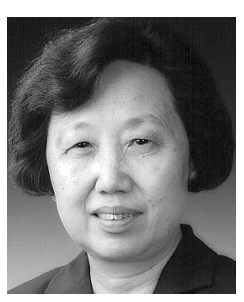

Shangkai Gao graduated from the Department of Electrical Engineering of Tsinghua University in 1970 and received the M.E. degree in biomedical engineering in 1982 from the same department of Tsinghua University, Beijing, China. She is currently a professor of the Department of Biomedical Engineering, Tsinghua University. She is an associate editor of IEEE Transactions on Biomedical Engineering and IEEE Transactions on Neural System and Rehabilitation Engineering. Her research interests include biomedical signal processing and medical ultrasound.

Address for Correspondence: Shangkai Gao, Department of Biomedical Engineering, School of Medicine, Tsinghua University, Beijing 100084 China. E-mail: gsk-dea@tsinghua.edu.cn.

\section{References}

[1] J. R. Wolpaw, N. Birbaumer, D. J. McFarland, G. Pfurtscheller, and T. M. Vaughan, "Brain-computer interfaces for communication and control," Clin. Neurophysiol., vol. 113, no. 2, pp. 767-791, 2002.
[2] M. A. Lebedev and M. A. L. Nicolelis, "Brain-machine interfaces: past, present and future," Trends Neurosci., vol. 29, no. 9, pp. 536-546, 2006.

[3] N. Birbaumer, "Brain-computer-interface research: Coming of age," Clin. Neurophysiol., vol. 117, no. 3, pp. 479-483, 2006.

[4] J. J. Vidal, "Real-time detection of brain events in EEG," Proc. IEEE, vol. 65 , no. 5 , pp. $633-641,1977$.

[5] E. E. Sutter, "The brain response interface: communication through visuallyinduced electrical brain response," J. Microcomput. Appl., vol. 15, no. 1, pp. 31-45, 1992.

[6] M. Middendorf, G. McMillan, G. Calhoun, and K. S. Jones, "Brain-computer interfaces based on the steady-state visual-evoked response," IEEE Trans. Rehabil. Eng., vol. 8, no. 2, pp. 211-214, 2000.

[7] M. Cheng, X. R. Gao, S. G. Gao, and D. F. Xu, "Design and implementation of a brain-computer interface with high transfer rates," IEEE Trans. Biomed. Eng., vol. 49, no. 10, pp. 1181-1186, 2002.

[8] X. Gao, D. Xu, M. Cheng, and S. Gao, "A BCI-based environmental controller for the motion-disabled," IEEE Trans. Neural Syst. Rehab. Eng., vol. 11, no. 2, pp. 137-140, 2003.

[9] Y. Wang, R. Wang, X. Gao, B. Hong, and S. Gao, "A practical VEP-based brain-computer interface," IEEE Trans. Neural Syst. Rehab. Eng., vol. 14, no. 2 , pp. 234-239, 2006.

[10] E. C. Lalor, S. P. Kelly, C. Finucane, R. Burke, R. Smith, R. B. Reilly, and G. McDarby, "Steady-state VEP-based brain-computer interface control in an immersive 3D gaming environment," EURASIP J. Appl. Signal Process. vol. 2005, no. 19 , pp. 3156-3164, 2005.

[11] G. R. Muller-Putz, R. Scherer, C. Brauneis, and C. Pfurtscheller, "Steadystate visual evoked potential (SSVEP)-based communication: impact of harmonic frequency components," J. Neural Eng., vol. 2, no. 4, pp. 123-130, 2005.

[12] S. P. Kelly, E. C. Lalor, C. Finucane, G. McDarby, and R. B. Reilly, "Visual spatial attention control in an independent brain-computer interface," IEEE Trans. Biomed. Eng., vol. 52, no. 9, pp. 1588-1596, 2005.

[13] L. J. Trejo, R. Rosipal, and B. Matthews, "Brain-computer interfaces for 1-D and 2-D cursor control: Designs using volitional control of the EEG spectrum or steady-state visual evoked potentials," IEEE Trans. Neural Syst. Rehab. Eng., vol. 14 , no. 2 , pp. 225-229, 2006.

[14] T. Kluge and M. Hartmann, "Phase coherent detection of steady-state evoked potentials: experimental results and application to brain-computer interfaces," in Proc. 3rd Int. IEEE EMBS Neural Engineering Conf., 2007, pp. 425-429.

[15] D. Regan, Human brain electrophysiology: evoked potentials and evoked magnetic fields in science and medicine. New York: Elsevier, 1989.

[16] S. T. Morgan, J. C. Hansen, and S. A. Hillyard, "Selective attention to stimulus location modulates the steady-state visual evoked potential," Proc. Nat. Acad. Sci., vol. 93, no. 10, pp. 4770-4774, 1996.

[17] Y. Q. Chen, A. K. Seth, J. A. Gally, and G. M. Edelman, "The power of human brain magnetoencephalographic signals can be modulated up or down by changes in an attentive visual task," Proc. Nat. Acad. Sci., vol. 100, no. 6, pp. 3501-3506, 2003.

[18] M. M. Muller, S. Andersen, N. J. Trujillo, P. Valdes-Sosa, P. Malinowski, and S. A. Hillyard, "Feature-selective attention enhances color signals in early visual areas of the human brain," Proc. Nat. Acad. Sci., vol. 103, no. 38, pp. 14250-14254, 2006.

[19] S. G. Mason, A. Bashashati, M. Fatourechi, K. F. Navarro, and G. E. Birch, "A comprehensive survey of brain interface technology designs," Ann. Biomed. Eng., vol. 35, no. 2, pp. 137-169, 2007.

[20] N. Birbaumer, "Breaking the silence: Brain-computer interfaces (BCI) for communication and motor control," Psychophysiology, vol. 43, no. 6, pp. 517$532,2006$.

[21] A. C. MettingVanRijn, A. P. Kuiper, T. E. Dankers, and C. A. Grimbergen, "Low-cost active electrode improves the resolution in biopotential recordings," in Proc. 18th Int. IEEE EMBS Conf., 1996, pp. 101-102.

[22] C. Jia, H. Xu, B. Hong, X. Gao, and S. Gao, "A human computer interface using SSVEP-based BCI technology," Lecture Notes in Computer Science, vol. 4565, pp. 113-119, 2007.

[23] T. M. S. Mukesh, V. Jaganathan, and M. R. Reddy, "A novel multiple frequency stimulation method for steady state VEP based brain computer interfaces," Physiol. Meas., vol. 27, no. 1, pp. 61-71, 2006.

[24] A. Materka and M. Byczuk, "Alternate half-field stimulation technique for SSVEP-based brain-computer interfaces," Electron. Lett., vol. 42, no. 6, pp. 321322, 2006.

[25] C. Fonseca, J. P. S. Cunha, R. E. Martins, V. M. Ferreira, J. P. M. de Sa M. A. Barbosa, and A. Martins da Silva, "A novel dry active electrode for EEG recording," IEEE Trans. Biomed. Eng., vol. 54, no. 1, pp. 162-165, 2007.

[26] R. Matthews, N. McDonald, H. Anumula, J. Woodward, P. Turner M. Steindorf, K. Chang, and J. Pendleton, "Novel hybrid bioelectrodes for ambulatory zero-prep EEG measurements using multi-channel wireless EEG system," Lecture Notes in Computer Science, vol. 4565, pp. 137-146, 2007.

[27] F. Popescu, S. Fazli, Y. Badower, B. Blankertz, and K. Müller, "Single trial classification of motor imagination using 6 dry EEG electrodes," PLOS ONE, vol. 2, no. 7, p. e637, 2007.

[28] O. Friman, I. Volosyak, and A. Graser, "Multiple channel detection of steady-state visual evoked potentials for brain-computer interfaces," IEEE Trans. Biomed. Eng., vol. 54, no. 4, pp. 742-750, 2007.

[29] Z. Lin, C. Zhang, W. Wu, and X. Gao, "Frequency recognition based on canonical correlation analysis for SSVEP-based BCIs," IEEE Trans. Biomed. Eng., vol. 53, no. 12, pp. 2610-2614, 2006. 